\title{
Growth Factors in the Blood Serum of Patients with Infection Complications of Polytrauma
}

\author{
Luneva SN, Klyushin NM, Dolganova TI, Matveeva EL* and Leonchuk DS \\ Federal State Budgetary Institution, Russian Ilizarov Scientific Centre, Restorative Traumatology and \\ Orthopaedics, Ministry of Health of the Russian Federation, Russia
}

\begin{abstract}
Purpose: Molecular aspects of organism functioning in ostemyelitis have not received an in-depth study. Therefore, the purpose of the work is to study the content of angiogenic factors in the blood serum in the patients with high-energy trauma.

Methods: 36 patients after high-energy trauma have been included in the study. The patients were divided into 2 groups. The first group comprised patients without infection complications and the second one included individuals with infection complications developed during treatment. Immune-enzyme study of vascular growth factors was performed using Thermofisher Company (USA) equipment complex and diagnostical sets of the following companies: $R$ \& D Systems (Ang 1, Ang 2, PDGF-AB, PDGF-BB), Invitrogen (EGF, VEGF), Ray Biotech (MSP); Biomerika Inc. (Calcitonin), USA, and Immunodiagnostic (BSP), Germany. A Hitachi Hi Vision Avius scanner was used to carry out ultrasonic tests of blood vessels in soft tissues and periosteum in the regenerate area (defect).
\end{abstract}

Results: The concentration of Ang-2 and VEGF in the 2 nd group was decreased for $60 \%$ compared to the concentration in the individuals without infection complications.

The serum concentration of EGF in individuals with complications after high energy trauma was 1.6 times higher than the same in people without infection complications after injuries.

Conclusion: Available data make possible to presume that the growth factors, the subjects of the study, can serve as additional diagnostic and prognostic markers of infection complications after high-energy trauma.

Keywords

High energy trauma, Growth factors, Connective tissue, Osteomyelitis

\section{Introduction}

Currently Ilizarov transosseous osteosynthesis technique is widely used in orthopedics, primarily for addressing severe injuries, their consequences and complications [1].

Molecular aspects of organism functioning in ostemyelitishave not received an in-depth study. A number of papers describe research on growth factors in osteosynthesis. In particular, it is indicated that bone regeneration is accompanied by growing concentrations of angiogenic growth factors in blood serum [2].

Ang-1, Ang-2 and EGF turn out to be involved in reparative bone regeneration [3].

It is well known that chronic osteomyelitis is accompanied by marked disorders of vascular system of the same segment [4], and it is believed that primary vascular disorders very often become the causes for acute and chronic infections of the bone system [5].
Vascular factors happen to be basically involved in bone regeneration as well as in cartilage transformation into the bone (endochondral ossification) in distractional regenerate formation.

Publications contain no information on concentrations, dynamics of growth factors data in orthopedic treatment of severe high energy injuries and their impact during chronic

*Corresponding author: Matveeva EL, Federal State Budgetary Institution, Russian Ilizarov Scientific Centre, Restorative Traumatology and Orthopaedics, Ministry of Health of the Russian Federation, Russia

Accepted: February 01, 2021

Published online: February 03, 2021

Citation: Luneva SN, Klyushin NM, Dolganova TI, et al. (2021) Growth Factors in the Blood Serum of Patients with Infection Complications of Polytrauma. J Orthop Surg Tech 4(1): 231-236 
Citation: Luneva SN, Klyushin NM, Dolganova TI, et al. (2021) Growth Factors in the Blood Serum of Patients with Infection Complications of Polytrauma. J Orthop Surg Tech 4(1): 231-236

infection development after surgical treatment, particularly in Ilizarov technique applications.

In general, evaluation of the available data shows that there is clear picture of the growth factors spectrum produced in transosseous distractional osteosynthesis, and there are no papers focusing on comparative analyses of the growth factors changes depending on the pathology of the locomotor system.

The purpose of this study is to investigate the content of angiogenic factors in the blood serum of patients with polytrauma.

\section{Materials and Methods}

The work analyzes angiogenic growth factors concentrations changes in blood serum of the patients with injuries of locomotor system complicated by infection complications. 36 patients after high-energy trauma have been included in the study. All patient under went treatment in a trauma center of the $1^{\text {st }}$ level. The patients with infection complications were referred to Infection Osteology Department of the Russian Ilizarov Scientific Centre for inpatient treatment.

The patients were divided into 2 groups. The first group comprised patients without infection complications and the second one included individuals with infection complications developed during treatment. The age in both groups ranged from 18 to 60 years. All patients referred to working age population.

The study started on the $3^{\text {rd }}$ day after operations to predict the infection complications development.

The blood serum indices of the patients were compared with the values obtained from a control group of 20 subjectively healthy people aged from 20 to 45-years.

The first group included 25 cases, i.e. sixteen (64\%) males and nine females (36\%). The second group had 11 individuals including seven (64\%) males and four (365) females.

Immune-enzyme study of vascular growth factors was performed using Thermofisher Company (USA) equipment

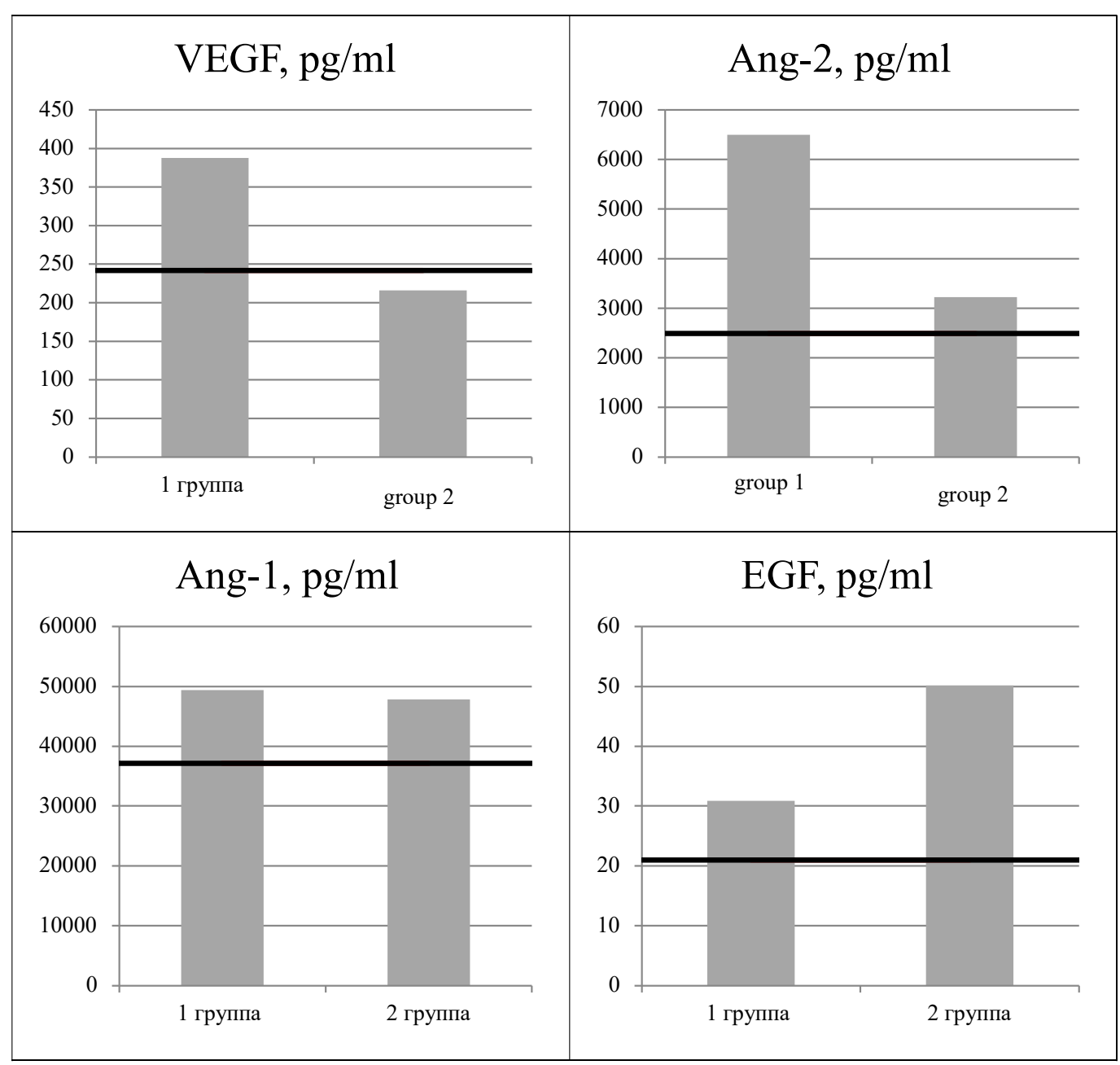

Figure 1: Dynamics of quotients/indices of vascular growth factors in blood serum in patients suffering from high-energy trauma as they were treated by transosseousosteosynthesis.

Note: Ang-1and Ang-2-angiopoietin 1 and 2; EGF: Epidermal Growth Factor; VEGF: Vascular Endothelial Growth Factor; The horizontal line represents the median of the values obtained from the control group

*- statistically significant difference between the groups, $p \leq 0.05$. 
Citation: Luneva SN, Klyushin NM, Dolganova TI, et al. (2021) Growth Factors in the Blood Serum of Patients with Infection Complications of Polytrauma. J Orthop Surg Tech 4(1): 231-236

complex and diagnostical sets of the following companies: R \& D Systems (Ang 1-angiopoetin 1, Ang 2-angiopoetin 2, PDGF-AB, PDGF-BB -platelet-derived growth factors); Invitrogen (EGF-epidermal growth factor; VEGF-vasculo endothelial growth factor) ; Ray Biotech (MSP- Macrophage stimulating protein); Biomerika Inc. (Calcitonin) (USA) and Immunodiagnostic (BSP-Bone sialoprotein), Germany.

Ultrasonic tests of blood vessels in the soft tissues and periosteum in the regenerate area (defect site) was carried out using "Hitachi" Hi Vision Avius scanner. Multifrequency linear sensor with a frequency of 5-12 $\mathrm{MHz}$ in B mode using colour flow and power Doppler mapping and Doppler spectrum analysis of vascular blood flow was applied.

Microcirculation in the tissues was evaluated using laser Doppler flowmetry (LDF) in BLF21 device of Transonic Sistems Inc. Local ischemic test was done by placing occlusive cuffs in tibia.

The following calculations were performed:

Peak blood flow index (PBI \% = CFpeak/CFrest ${ }^{*} 100 \%$ );

$\Delta t$, sec-time interval from occlusion termination to maximum capillary blood flow increase;

Capillary blood flow semi-restoration $\left(T_{1 / 2}, \sec \right)$;

And reactive hyperemia duration $(T, \mathrm{sec})$.

The Committee on Ethics at the Russian Ilizarov Scientific Centre "RTO" has granted permission to conduct this research. The researchers closely observed the Ethical Standards of the Declaration of Helsinki adopted by the World Medical Association "Ethical Principles for Medical Research Involving Human Subjects", amendments made in 2000.

The results of the research study were processed using the variational statistics technique for small samples and assuming a significance level of $p \leq 0.05$.

Statistical significance of deviations between two unrelated samples was defined by the Wilcoxon W-criterion, Mann-Whitney and Dann criteria [6].

The findings are presented as medians and lower and upper quartiles.

\section{Results}

Growth factor concentration values in the blood serum of all the patients showed no differences related to sex.

No significant differences in Ang-1 concentrations were found in the blood serum in the patients (Figure 1) who sustained high-energy injury and the difference between the groups was merely $7 \%$.

The concentration of Ang- 2 and VEGF in the $2^{\text {nd }}$ group was decreased for $60 \%$ compared to the concentration in the individuals without infection complications; and $p=0.034$.

The serum concentration of EGF in individuals with complications after high energy trauma was 1.6 times higher than the same in people without infection complications after injuries.

The study of the growth factors, which included plate- let-derived growth factor PDGF-AB and PDGF-BB in both groups (Figure 2 ) did not reveal any significant differences between groups. In addition, even concentration of calcitonin and bone sialoprotein and macrophage of stimulating protein did not differ between the groups.

Our data on dominant changes in the concentrations of vascular growth factors were confirmed by the results of Doppler scanning. UDS (Figure 3) showed that the patients with high energy injuries and without complications had enhanced colour mapping of the arterial and venous components in the cortical zone of the defect and adjacent soft tissues. The patients with infection revealed during treatment showed poor vascular net in the cortical zone and adjacent soft tissues.

To assess miocrocirculation in skin tissues (Table 1) data were obtained applying Doppler flowmetry.

Indications describing CF rest and CF peak do not have significant differences in the groups since spare capacities of the vascular bed are preserved. Anyway, the 3-minute ischemic test appear to reveal vasoconstriction, reduced spare capacities of the organism, metabolic disorder and malfunctioning vascular endothelium, i.e. the index of the blood flow peak falls dramatically, the interval from occlusion removal time to the maximum capillary blood flow increase and the length of reactive hyperemia duration that can be interpreted as manifestation of vasoconstriction [7].

\section{Discussion}

Infection processes are the most challenging complications of high-energy injuries. Their developments are directly related to severity of injury, volume of operative in vasion and prevention measures [8]. But the question remains quite persistent why being under identical conditions and having same scope of medical aid infection complications develop in some patients. It is obvious that development of infection processes are likely to be dependent on a patient's organism, its immunity, concomitant diseases and resistance to infection $[9,10]$. Currently this question remains poorly known; neither predictors nor mechanisms of development of these complications have not been clarified [11]. Maintainance of normal blood flow appears is essential for restoration of structural and functional cells injuris after high-energy trauma accompanied by hypoxia and tissue ischemia [12-15]. VEGF, the heterodimeric glycoprotein growth factor, is known to be responsible for revascularization and angiogenesis. Moreover, being a mitogen for epithelial cells in blood vessels VEGF is capable to increase vascular permeability. It is indicated that combined cation of VEGF and Ang-1 in blood serum promotestissue vascularization and witnesses the persistent infection that is a precursor of morphological changes and leads to progressing degeneration of the connective tissue.

For results evaluation of this study, particular attention should be given to synergistic action of Ang-2 and VEGF factors that are observed in the patients with inflection developed in post-operative time. It can be presumed that on the background of general vascular destruction of the affected segment the reduction of concentrations of these factors leads to higher permeability vascular walls for infection 


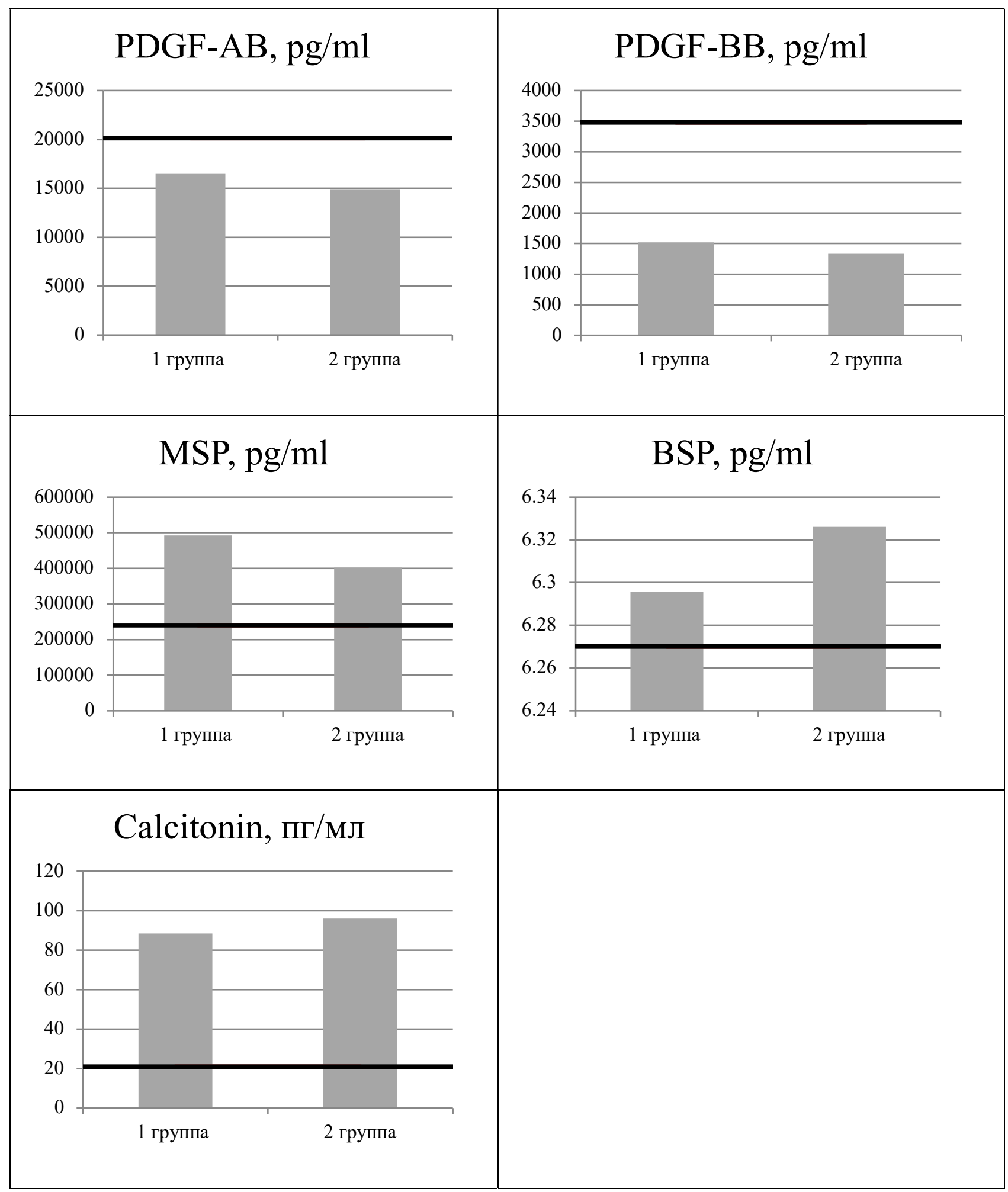

Figure 2: Dynamics of quotients/indices of regulatory factors in blood serum in patients after high-energy trauma by transosseous osteosynthesis.

Note: PDGF-AB, PDGF-BB-Platelet Derived Growth Factors

MSP: Macrophage Stimulating Protein; BSP: Bone Sialoprotein; The horizontal line represents the median of the values obtained from the control group

*- statistically significant difference between the groups, $\mathrm{p} \leq 0.05$. 
Citation: Luneva SN, Klyushin NM, Dolganova TI, et al. (2021) Growth Factors in the Blood Serum of Patients with Infection Complications of Polytrauma. J Orthop Surg Tech 4(1): 231-236

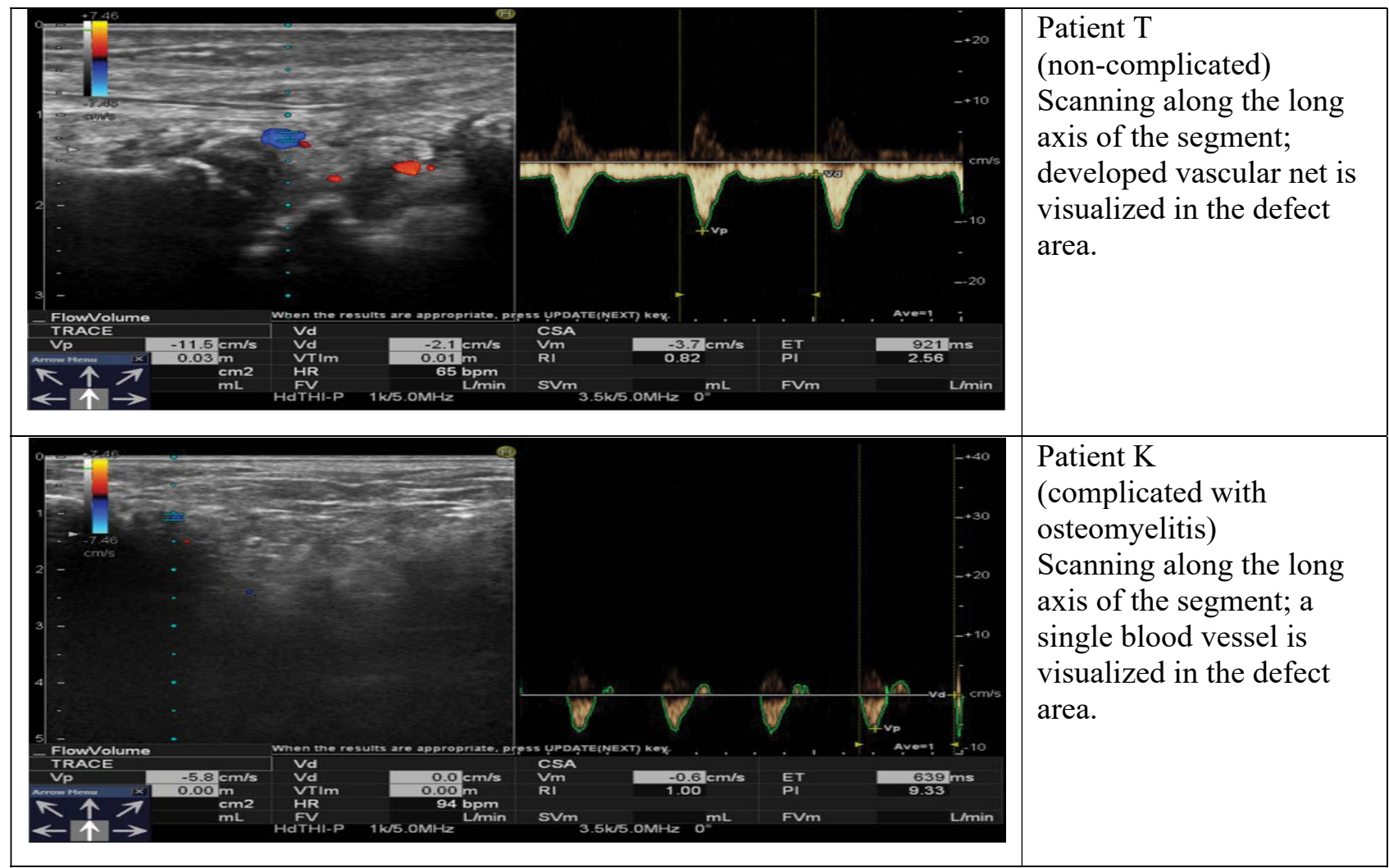

Figure 3: Colour mapping and UDS of arterioles in the cortical zone and adjacent soft tissues.

Table 1: Indices of microcirculation in skin tissue in patients with high-energy traumata treated by transosseous osteosynthes is.

\begin{tabular}{|l|l|l|}
\hline Indices & Group 1 & Group 2 \\
\hline $\mathrm{CF}$ rest. & $1.8(1.5 \div 2.1)$ & $2.0(1.8 \div 2.9)$ \\
\hline $\mathrm{CF}$ peak & $9.4(6.4 \div 11.3)$ & $7.0(4.5 \div 9.3)$ \\
\hline $\mathrm{PBI}, \%$ & $575(410 \div 625)$ & $341(230 \div 400)^{*}$ \\
\hline$\Delta \mathrm{t}, \mathrm{sec}$ & $20(15 \div 35)$ & $10(5 \div 12)^{*}$ \\
\hline $\mathrm{T}_{1 / 2,} \mathrm{sec}$ & $35(25 \div 40)$ & $25(20 \div 35)$ \\
\hline $\mathrm{T}, \mathrm{sec}$ & $120(90 \div 300)$ & $60(40 \div 75)^{*}$ \\
\hline
\end{tabular}

Note: CF rest, ph unit-capillary blood flow at rest; CF peak, ph unit-peak capillary blood flow after 3-minutes ischemia; PBI, peak blood flow index \%; $\Delta \mathrm{t}$, sec-time interval from occlusion removal to maximum capillary blood flow increase; $T_{1 / 2}$, sec-period of capillary blood flow semi-restoration; $T$, sec-reactive hyperemia duration.

* -statistically significant difference between the groups, $p \leq 0.05$.

agents and increase of the number of pyogenic organisms leading to changes in the metabolism in the affected zone and in other organs and tissues accompanied with functional changes in the life support system.

EGF, the epidermal growth factor, stimulating cellular growth, promotes regulation and differentiation in the epithelial lining and new vessels formation. Patients with infection complications demonstrate increased concentrations of this factor in peripheral blood. On the background of poor vascular net in the cortical area and adjacent soft tissues is reasonable to presume that its growth is compensatory.
In addition to the above we did not state the statistically significant changes in the concentrations of platelet-derived growth factors (PDGF-AB, PDGF-BB), calcitonina, bone sialoprotein (BSP), and stimulating protein macrophage.

\section{Conclusion}

The changes registered in all examined patients indicate a moderate extent of vascular system restoration. The patients with chronic posttraumatic osteomyelitis showed an imbalance of angiogenic growth factors in blood serum.

Available data make possible to presume that the growth factors, the subjects of the study, can serve as additional diagnostic and prognostic markers of infection complications after high-energy trauma.

\section{Conflict of Interests}

The authors declare that there is no conflict of interests in the work.

\section{References}

1. Aktuglu K, Erol K, Vahabi A (2019) Ilizarov bone transport and treatment of critical-sized tibial bone defects: A narrative review. J Orthop Traumatol 20: 22.

2. Hankenson KD, Dishowitz M, Gray C, et al. (2015) Angiogenesis in bone regeneration. Injury 42: 556-561.

3. Zhang M, Yu W, Niibe K, et al. (2018) The effects of platelet-derived growth factor-bb on bone marrow stromal cell-mediated vascularized bone regeneration. Stem Cells Int 2018: 3272098. 
4. Cheng L, Yang X, Chen T, et al. (2017) Peroneal artery perforator flap for the treatment of chronic lower extremity wounds. J Orthop Surg Res 12: 170.

5. Arnold JF (2018) Is there adequate perfusion for healing? What routine noninvasive vascular studies are missing? Wounds 30 E89-E92.

6. Stogov MV, Luneva SN, Novikov KI (2013) Growth factors in human serum during operative tibial lengthening with the ilizarov method. Journal of Orthopaedic Research 31: 1966-1970.

7. Kochetkov AV, Gudilov MS (2015) Clinical and laboratory diagnosis and monitoring of septic complications after operations. NovostiKhirurgii 23: 105-111.

8. Horst K, Hildebrand F, Pfeifer R, et al. (2015) Plate osteosynthesis versus hemiarthroplasty in proximal humerus fractures--does routine screening of systemic inflammatory biomarkers makes sense? Eur J Med Res 20: 5.

9. Guo H, Zhou H, Lu J, et al. (2016) Vascular endothelial growth factor: An attractive target in the treatment of hypoxic/ischemic brain injury. Neural regeneration research 11: 174-179.
10. Blanco R, Gerhardt H (2013) VEGF and notch in tip and stalk cell selection. Cold Spring Harb Perspect Med 3: a006569.

11. Ivanov AN, Grechikhin AA, Norkin IA, et al. (2014) Methods of diagnosing the endothelial function. Regionarnoe Krovoobrashcheniei Mikrotsirkuliatsiia 13 4: 4-11.

12. Liu TL, Maio JC, Sheng WH, et al. (2010) Cytocompatibility of regenerated silk fibroin film: A medical biomaterial applicable to wound healing. J Zhejiang Univ Sci B 11: 10-16.

13. Akwii RG, Sajib MS, Zahra FT, et al. (2019) Role of angiopoietin-2 in vascular physiology and pathophysiology. Cells 8: 471.

14. Fawcett LB (1998) Limb reduction defects in the A/J mouse strain associated with maternal blood loss. Teratology 58: 183-189.

15. Yin P, Ji Q, Li T, et al. (2015) A systematic review and meta-analysis of ilizarov methods in the treatment of infected nonunion of tibia and femur. PLoS One 10: e0141973. 